\title{
A method for determining gas-hydrate or free-gas saturation of porous media from seismic measurements
}

\author{
Matthias Zillmer ${ }^{1}$
}

\begin{abstract}
The occurrence of gas hydrate or free gas in a porous medium changes the medium's elastic properties. Explicit formulas for gas-hydrate or free-gas saturation of pore space on the basis of the Frenkel-Gassmann equations describe the elastic moduli and seismic velocities of a porous medium for low frequencies. A key assumption of the model is that either gas hydrate or free gas is present in the pore space in addition to water. Under this assumption, the method uses measured P- and S-wave velocities and bulk density along with estimates of the moduli and densities of the solid and fluid phases present to determine whether gas or hydrate is present. The method then determines the saturation level of either the gas or the hydrate. I apply the method to published velocity and density data from seismic studies at the antarctic Shetland margin and at the Storegga slide, offshore Norway, and to borehole log and core data from Ocean Drilling Program (ODP) Leg 164 at Blake Ridge, offshore South Carolina. A sensitivity analysis reveals that the standard deviations of the gas-hydrate and free-gas saturations reach $30 \%-70 \%$ of the saturations if the standard deviations of the $\mathrm{P}$ - and $\mathrm{S}$-wave velocities and of the bulk density are $50 \mathrm{~m} / \mathrm{s}$ and $0.05 \mathrm{~g} / \mathrm{cm}^{3}$, respectively. I conclude that a reliable quantification of gas hydrate and free gas can be achieved by seismic methods only if the seismic velocities and bulk density of the medium are determined with high accuracy from the measured data.
\end{abstract}

\section{INTRODUCTION}

Gas hydrates are ice-like solid substances composed of water molecules that enclose gas molecules. They occur in permafrost areas and in marine sediments at continental margins in the first few hundred meters below the sea floor (bsf), where appropriate temperature and pressure-stability conditions are fulfilled and free methane gas is present (Kaplan, 1974; Cox, 1983; Sloan et al., 1994; Max, 2003). The elastic properties of gas-hydrate- and freegas-bearing sediments differ from those of fully water-saturated sediments, and these differences can be detected with seismic waves. Gas hydrate as a suspension in pore water decreases the compressibility of the pore fluid, while hydrate as a part of the solid material reduces the porosity and may cement the sediment grains, thus stiffening the sediment frame (Ecker et al., 1998, 2000; Helgerud et al., 1999). Free gas increases the compressibility of the pore fluid. Domenico (1976) shows that a small amount of free gas in the pore space strongly reduces the P-wave velocity. This effect is more pronounced for a homogeneous distribution of gas and water, and it is weaker in the case of patchy distribution (Dvorkin and Nur, 1998).

Different methods have been applied to determine the amount of gas hydrate and free gas in marine sediments based on seismic measurements. A simple formula is Wyllie's time average equation, which represents an average of the inverse P-wave velocities of the sediment constituents. For fine-structured media and large wavelengths, it is more adequate to average the inverse elastic moduli (Backus, 1962). Wood's equation as the average of the inverse $\mathrm{P}$-wave moduli is applicable to a mixture of two fluids, but the stiffness of the sediment frame is neglected. Lee et al. (1996) suggest using the sum of Wyllie's and Wood's equations with empirical scaling factors, but the procedure lacks a theoretical foundation. The Frenkel-Gassmann and Geertsma-Smit equations describe the elastic moduli and seismic velocities of a porous medium that consists of a solid phase and a fluid phase (Frenkel, 1944; Gassmann, 1951; Geertsma and Smit, 1961). They can be combined with models that take into account the effect of gas hydrate and free gas (Domenico, 1976; Ecker et al., 1998, 2000; Helgerud et al., 1999; Tinivella, 1999). Following this approach, Zillmer et al. (2005a, b) derive formulas for the saturations in explicit form and apply them to marine seismic data. In the present article I derive a different form of these equations, where porosity does not appear as an independent variable and where the dry medium is represented by the ratio of $\mathrm{P}$ - to $\mathrm{S}$-wave velocity, which is nearly constant.

Manuscript received by the Editor February 28, 2005; revised manuscript received September 29, 2005; published online May 22, 2006.

${ }^{1}$ IFM-GEOMAR, Leibniz Institute of Marine Sciences, Wischhofstraße 1-3, D-24148 Kiel, Germany. E-mail: mzillmer@ifm-geomar.de. (c) 2006 Society of Exploration Geophysicists. All rights reserved. 
If the Frenkel-Gassmann equations are used to quantify gas hydrate and free gas, then four problems arise. First, the equations connect the elastic moduli of the wet (saturated) and dry (unsaturated) medium, which is also called the frame, matrix, or skeleton. The wet moduli can be determined in situ from seismic measurements, unlike the dry moduli. Using different theoretical or empirical functions for the dry bulk modulus can lead to very different gas-hydrate-saturation estimates (Zillmer et al., 2005a). The reason for this effect is that gas-hydrate-bearing sediments often have porosities near the critical porosity, which describes the transition from the suspension domain to the consolidated-rock domain (Nur et al., 1998). In this region the elastic moduli of the dry medium vary strongly with porosity, especially if gas hydrate cements the sediment grains. According to Frenkel and Gassmann, the dry shear modulus equals the wet shear modulus; thus, it can be obtained by measuring S-wave velocity and bulk density. Appendix B shows that the ratio of the dry bulk modulus to the shear modulus is nearly constant for some theoretical models, a property reported in several experimental studies (e.g., Dvorkin and Yin, 1995; Priest et al., 2005). As a consequence, the dry bulk modulus can also be estimated from the measured S-wave velocity and bulk density.

Second, S-wave velocity and bulk density are often not derived from measured data but are computed for the model of an aggregate of spheres (Dvorkin et al., 1999); sometimes empirical average curves are used (e.g., Hamilton, 1971, 1976). I show that a reliable estimate for the saturation can only be obtained if these variables are known with high accuracy, which implies they should be obtained directly from the measured data to be characteristic of the investigated medium.

Third, it is commonly accepted that the Frenkel-Gassmann equations give useful results for low frequencies (Mavko et al., 1998). However, they are valid only for one solid phase, while marine sediments consist of several minerals with different elastic constants. Jacobsen et al. (2000) have developed a multiphase theory based on the self-consistent approximation (SCA) and the differential effective medium (DEM) theory. They apply their theory to data from the Blake Ridge (offshore South Carolina) and obtain a peak value of 9 vol \% gas-hydrate saturation, which corresponds to $16 \%$ of the pore space at $55 \%$ porosity. Following Dvorkin et al. (1999), I assume the Frenkel-Gassmann equations can be used with an effective average bulk modulus and density for the solid phase. Using this approach, I obtain a result similar to that of Jacobsen et al. (2000) for the Blake Ridge sediments. Chand et al. (2004) obtain similar results for the gas-hydrate saturation of the Blake Ridge sediments by using Jacobsen's method and by using the Frenkel-Gassmann equations (the three-phase effective medium, or TPEM, method). They use P- and S-wave velocity separately to estimate gas-hydrate saturation. I combine the two Frenkel-Gassmann equations for the bulk and shear moduli to eliminate the dry frame moduli from the problem. Thus, I obtain only one result for the saturation, which is consistent with both velocities.

Finally, porosity is an important parameter that cannot be measured directly with seismic methods. I derive porosity from bulk density, which can be determined with an amplitude variation with offset (AVO) analysis or by advanced inverse methods (e.g., Beylkin and Burridge, 1990). Zillmer et al. (2005a) use the density of a fully water-saturated medium to approximate the densities of gashydrate and free-gas-bearing media. The formulas presented here do not require this approximation.

This study applies the method to seismic and borehole data, for which information about $\mathrm{P}$ - and S-wave velocities and bulk density has been published: (1) the seismic studies of Tinivella and Accaino (2000) from the antarctic Shetland margin and of Andreassen et al. (2003) from the Storegga slide, offshore Norway, and (2) the borehole log and core data from Blake Ridge acquired during ODP Leg 164 (Paull et al., 1996, 2000) and investigated by Guerin et al. (1999). I discuss the sensitivity of the results with respect to inaccuracies of the measured input variables.

\section{GAS-HYDRATE AND FREE-GAS SATURATION}

The elastic moduli of a wet (saturated) and unsaturated dry porous medium are connected by the equations

$$
\mu=\mu_{\mathrm{dry}}
$$

and

$$
\frac{\sigma^{2}}{K-K_{\text {dry }}}=\frac{\phi}{K_{f}}+\frac{\sigma-\phi}{K_{s}},
$$

where

$$
\sigma=1-\frac{K_{\mathrm{dry}}}{K_{s}}
$$

Here, $\mu$ and $\mu_{\text {dry }}$ are the shear moduli of the wet and dry medium; $K, K_{\mathrm{dry}}, K_{f}$, and $K_{s}$ are the bulk moduli of the wet and dry medium of the fluid and solid phases, respectively; and $\phi$ is the porosity. (Major variables used in the text are listed in Table 1.) The equations are named after Gassmann (1951), but Frenkel (1944) showed earlier that they can be used to obtain wave-propagation velocities in a porous medium. Pride and Garambois (2005) point out an error in Frenkel's calculations that prevented him from obtaining the correct final form of equation 2 . The result also follows as a limiting case of Biot's theory for low frequency (e.g., Biot and Willis, 1957; Geertsma and Smit, 1961; Stoll, 1991; Carcione, 2001). The wet moduli can be obtained by measuring P- and Swave velocities and bulk density, but the dry moduli cannot be measured in situ and vary strongly with porosity and pressure.

The influence of free gas and gas hydrate on the elastic properties of a medium is discussed by Ecker et al. $(1998,2000)$ and Helgerud et al. (1999). Free gas increases the compressibility of the pore fluid, while gas hydrate as a suspension in pore water has the opposite effect. Gas hydrate as a part of the solid reduces the porosity and stiffens the sediment frame. Zillmer et al. (2005a) show that the model of gas hydrate as a part of the solid leads to approximately 0.7 times smaller saturations than the model of hydrate as a part of the fluid. Ecker et al. (1998) present mechanical models where gas hydrate cements the sediment grains, leading to high elastic moduli of the dry sediment frame. Here, I assume that S-wave velocity and bulk density of the wet medium are obtained from measurements so that a cementation effect, which leads to a high shear modulus of the dry medium, is taken into account via equation 1. Guerin et al. (1999) discuss a model where gas hydrate and free gas are both present in the sediment. I restrict this study to the case that either free gas or gas hydrate is present. The derivations for the gas-hydrate and free-gas saturations are given in Appendix A, and the results are summarized in the following. 
If gas hydrate is a part of the solid phase, then the gas-hydrate saturation $S_{h}$ of the pore space is given by

$$
S_{h}=\frac{\left(\rho_{s}-\rho\right)\left(\frac{1}{K_{w}}-\frac{1}{K_{s}}\right)-\left(\rho_{s}-\rho_{w}\right) \sigma\left(\frac{\sigma}{M}-\frac{1}{K_{s}}\right)}{\left(\rho_{s}-\rho\right)\left(\frac{1}{K_{w}}-\frac{1}{K_{s}}\right)+\left(\rho_{w}-\rho_{h}\right) \sigma\left(\frac{\sigma}{M}-\frac{1}{K_{s}}\right)},
$$

where

$$
M=\rho\left(v_{p}^{2}-\gamma_{\mathrm{dry}}^{2} v_{s}^{2}\right)
$$

and

$$
\sigma=1-\frac{\left(\gamma_{\text {dry }}^{2}-\frac{4}{3}\right) \rho v_{s}^{2}}{K_{s}} .
$$

Here, $\gamma_{\text {dry }}$ is the $\mathrm{P}$ - to $\mathrm{S}$-wave velocity ratio of the dry frame.

If free gas is homogeneously distributed in the pore water (Do-

Table 1. Symbols used in the text. A lower index dry refers to the properties of the dry medium, while a lower index $c$ refers to the critical porosity of a random aggregate of spheres (see Appendix B).

\begin{tabular}{ll} 
Symbol & Definition \\
\hline$K$ & Bulk modulus \\
$K_{f}$ & Bulk modulus of the pore fluid (water, gas) \\
$K_{g}$ & (Adiabatic) bulk modulus of gas \\
$K_{w}$ & Bulk modulus of pore water \\
$K_{s}$ & Bulk modulus of solid material (sediment grains) \\
$M$ & Defined by equation 5 \\
$S$ & Saturation \\
$S_{g}$ & Free-gas saturation of the pore space \\
$S_{h}$ & Gas-hydrate saturation of the pore space \\
$v_{p}$ & P-wave velocity \\
$v_{s}$ & S-wave velocity \\
$\mu$ & Shear modulus \\
$\nu$ & Poisson's ratio \\
$\nu_{s}$ & Poisson's ratio of elastic spheres (Appendix B) \\
$\Omega$ & Reduced porosity of the gas-hydrate-bearing \\
$\gamma$ & Sediment \\
$\rho$ & Ratio of P- and S-wave velocity \\
$\rho_{g}$ & Bulk density \\
$\rho_{h}$ & Density of gas \\
$\rho_{s}$ & Density of gas hydrate \\
$\rho_{w}$ & Density of solid material (sediment grains) \\
$\sigma$ & Density of pore water \\
\hline & Defined by equation 6 \\
& Porosity \\
\hline &
\end{tabular}

menico, 1976), i.e., the same ratio of water and gas is present in all pores, then the free-gas saturation $S_{g}$ is given by

$$
S_{g}=\frac{\left(\rho_{s}-\rho_{w}\right) \sigma\left(\frac{\sigma}{M}-\frac{1}{K_{s}}\right)-\left(\rho_{s}-\rho\right)\left(\frac{1}{K_{w}}-\frac{1}{K_{s}}\right)}{\left(\rho_{s}-\rho\right)\left(\frac{1}{K_{g}}-\frac{1}{K_{w}}\right)-\left(\rho_{w}-\rho_{g}\right) \sigma\left(\frac{\sigma}{M}-\frac{1}{K_{s}}\right)} .
$$

For a patchy distribution of free gas and water (Dvorkin and Nur, 1998; Helgerud et al., 1999), the gas saturation is obtained by solving the quadratic equation

$$
A S_{g}^{2}+B S_{g}+C=0
$$

where the coefficients are given by

$$
\begin{aligned}
& A=\left(\rho_{w}-\rho_{g}\right)\left(\rho_{s}-\rho\right) \sigma^{2} \rho v_{p}^{2}\left(\frac{1}{K_{g}}-\frac{1}{K_{s}}\right) \\
& -\left(\rho_{w}-\rho_{g}\right)^{2} D \\
& B=\left(\rho_{s}-\rho_{w}\right)\left(\rho_{s}-\rho\right) \sigma^{2} \rho v_{p}^{2}\left(\frac{1}{K_{g}}-\frac{1}{K_{s}}\right) \\
& -\left(\rho_{s}-\rho_{w}\right)\left(\rho_{w}-\rho_{g}\right) 2 D+\left(\rho_{w}-\rho_{g}\right)\left(\rho_{s}-\rho\right) E, \\
& C=\left(\rho_{s}-\rho\right)^{2} \gamma_{\mathrm{dry}}^{2} \rho v_{s}^{2} M\left(\frac{1}{K_{g}}-\frac{1}{K_{s}}\right)\left(\frac{1}{K_{w}}-\frac{1}{K_{s}}\right)-\left(\rho_{s}\right. \\
& \left.-\rho_{w}\right)^{2} D+\left(\rho_{s}-\rho_{w}\right)\left(\rho_{s}-\rho\right) E, \\
& D=\sigma^{2}\left(1+\frac{\frac{4}{3} \rho v_{s}^{2}}{K_{s}}\right)\left[1-\frac{\rho\left(v_{p}^{2}-\frac{4}{3} v_{s}^{2}\right)}{K_{s}}\right], \\
& E=\sigma\left(M\left(1+\frac{\frac{4}{3} \rho v_{s}^{2}}{K_{s}}\right)\left(\frac{1}{K_{w}}-\frac{1}{K_{s}}\right)\right. \\
& \left.-\gamma_{\text {dry }}^{2} \rho v_{s}^{2}\left[1-\frac{\rho\left(v_{p}^{2}-\frac{4}{3} v_{s}^{2}\right)}{K_{s}}\right]\left(\frac{1}{K_{g}}-\frac{1}{K_{s}}\right)\right\} \text {. }
\end{aligned}
$$

A solution of this equation is accepted if the porosity computed with equation $\mathrm{A}-10$ is between 0 and 1 . For patchy distribution, the following approximate solution can be derived: 


$$
\begin{aligned}
S_{g} \approx & \left\{\sigma^{2}+\gamma_{\mathrm{dry}}^{2} \rho v_{s}^{2}\left[\frac{\sigma}{K_{s}}+\frac{\rho_{s}-\rho}{\rho_{s}-\rho_{w}}\left(\frac{1}{K_{g}}-\frac{1}{K_{s}}\right)\right]\right\} \\
& \times\left\{\sigma^{2}-M\left[\frac{\sigma}{K_{s}}+\frac{\rho_{s}-\rho}{\rho_{s}-\rho_{w}}\left(\frac{1}{K_{w}}-\frac{1}{K_{s}}\right)\right]\right\} \\
& \times\left\{\sigma^{2} \rho v_{p}^{2} \frac{\rho_{s}-\rho}{\rho_{s}-\rho_{w}}\left(\frac{1}{K_{g}}-\frac{1}{K_{w}}\right)\right\}
\end{aligned}
$$

Equations 4, 7, and 9 and one of the solutions of equation 8 have the same zeros, which describe the case of a fully water-saturated medium. Both $S_{h}$ and $S_{g}$ are to be computed. One of these values is always negative, which indicates this phase is not present. The positive value is the model's prediction for the saturation of either the hydrate or gas present. Again, if both gas and hydrate are present simultaneously in the rock sample, the model does not apply. Small values of $S_{g}$ and $S_{h}$ in combination with relatively large errors should be attributed to a fully water-saturated medium. The sensitivity of the saturation estimate with respect to inaccuracies of the input variables is investigated in Appendix C.

All quantities on the right-hand side of the formulas have a physical meaning. The $\mathrm{P}$ - and $\mathrm{S}$-wave velocities $v_{p}$ and $v_{s}$ and the bulk density $\rho$ can be obtained from traveltimes and amplitudes of seismic waves, from borehole logs, or from core measurements. The bulk moduli $K_{w}$ and $K_{g}$ and the densities $\rho_{w}$ and $\rho_{g}$ are functions of pressure, temperature, and salinity (Batzle and Wang, 1992; Mavko et al., 1998). Helgerud (2001) measures the gas-hydrate density $\rho_{h}$ in laboratory experiments. The Frenkel-Gassmann equations are strictly valid only for one solid phase, as are equations 4-9. I follow the approach of Dvorkin et al. (1999) and compute the bulk modulus of the solid phase $K_{s}$ by averaging the moduli of the sediment constituents. The precise value of $K_{s}$ is less important as long as $K_{s}^{-1}$ are by far the smallest compressibilities, which is the case for small gas-hydrate saturations. If the gashydrate saturation is very high, then equation 4 can be solved numerically with $K_{s}$ as a function of $S_{h}$.

The dry medium is described entirely by the P- to $\mathrm{S}$-wave velocity ratio $\gamma_{\text {dry }}$, and values for this ratio must be assumed. Theoretical results show that $\gamma_{\text {dry }}^{2}$ ranges between two and three for unconsolidated or slightly consolidated media and does not vary strongly with porosity, pressure, and mineralogy (Appendix B). Hamilton (1971) presents laboratory results from velocity measurements in clean sand samples, where $\gamma_{\text {dry }}^{2}$ is 1.9-2.6 for different pressures. Winkler (1983) obtains $\gamma_{\text {dry }}^{2}$ between 2.3 and 2.5 from velocity data for unconsolidated glass beads and for sandstone. Spencer et al. (1994) measure $\gamma_{\text {dry }}^{2}$ as 2.2-2.9 for a worldwide collection of unconsolidated sands and sandstones. Dvorkin and Yin (1995) present a value of 2.9 for epoxy-cemented glass beads, and Dvorkin and Nur (1998) report 2.2-2.4 for unconsolidated sands and 2.4-2.7 for cemented sandstone. Priest et al. (2005) perform laboratory experiments with gas-hydrate-bearing sand samples and obtain average values of 1.95 without cementation effect and 2.25 for gas-hydrate-cemented samples (Table 2 of Priest et al., 2005). The experimental results scatter in between two and three. I arbitrarily choose 2.4 for $\gamma_{\text {dry }}^{2}$ to compute the free-gas saturation in an uncemented medium, and I use 2.7 to compute the gas-hydrate saturation, where this latter value accounts for cementation effect.

The gas-hydrate saturation $S_{h}$ is shown in Figure 1 as a function of P- and S-wave velocities and bulk density. Densities of 1.6, 1.8, and $2.0 \mathrm{~g} / \mathrm{cm}^{3}$ correspond to porosities of $64 \%, 52 \%$, and $40 \%$, the typical interval for gas-hydrate-bearing marine sediments. High values of $S_{h}$ are obtained for high P-wave velocities, for low $\mathrm{S}$-wave velocities, and for low densities. This pattern mathematically is a consequence of the different signs in front of $\mathrm{P}$ - and $\mathrm{S}$-wave velocities in the formula for the bulk modulus (equation A-6). It is not a contradiction of the fact that $\mathrm{P}$ - and $\mathrm{S}$-wave velocities both increase with increasing gas-hydrate content. The gashydrate saturation strongly depends on all three parameters and cannot be estimated reliably only on the basis of measured P-wave velocities.

The free-gas saturation $S_{g}$ is shown in Figure 2 for a density of $1.8 \mathrm{~g} / \mathrm{cm}^{3}$ as a function of P- and S-wave velocities. The approximate solution (equation 9) gives results close to the exact solution of equation 8 . The terms proportional to $K_{g}^{-1}$ dominate equations 7 and 9 . It follows that the free-gas saturation is approximately linear proportional to the gas bulk modulus $K_{g}$ in the case of a homogeneous distribution, and it is nearly independent of $K_{g}$ for patchy distribution.

\section{APPLICATION TO SEISMIC AND BOREHOLE DATA}

The gas-hydrate and free-gas saturation curves of Figures 1 and 2 demonstrate that not only $\mathrm{P}$-wave velocity but also S-wave velocity and bulk density should be obtained from measurements to allow for reliable quantification. These variables were derived for
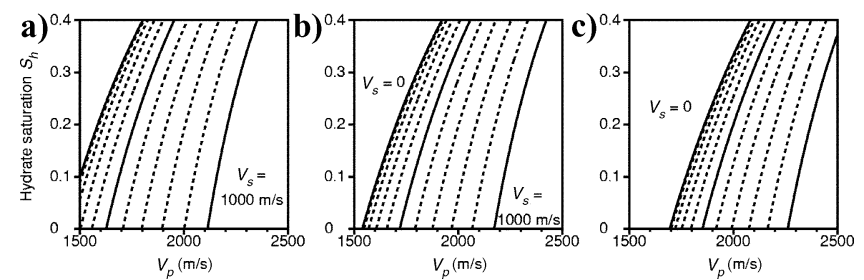

Figure 1. Gas-hydrate saturation $S_{h}$ as a function of $v_{p}, v_{s}$, and $\rho$. (a) $\rho=1.6 \mathrm{~g} / \mathrm{cm}^{3}, K_{w}=2.2 \mathrm{GPa}$; (b) $\rho=1.8 \mathrm{~g} / \mathrm{cm}^{3}, K_{w}=2.4$ $\mathrm{GPa}$; (c) $\rho=2.0 \mathrm{~g} / \mathrm{cm}^{3}, K_{w}=2.6 \mathrm{GPa}$. Densities of $1.6,1.8$, and $2.0 \mathrm{~g} / \mathrm{cm}^{3}$ correspond to porosities of about $64 \%, 52 \%$, and $40 \%$. The other parameters used are $K_{s}=30 \mathrm{GPa}, \rho_{w}=1.03 \mathrm{~g} / \mathrm{cm}^{3}, \rho_{s}$ $=2.65 \mathrm{~g} / \mathrm{cm}^{3}, \gamma_{\text {dry }}^{2}=2.7$. The $\mathrm{S}$-wave velocity is shown in increments of $100 \mathrm{~m} / \mathrm{s}(0,500$, and $1000 \mathrm{~m} / \mathrm{s}$ as solid lines $)$. In the limiting case $v_{s}=0$, the dry sediment is a suspension of grains in the pore water. In this case the Frenkel-Gassmann equation is reduced to the volumetric average of the inverse P-wave modulus of the solid and the pore water; the gas hydrate reduces the porosity.
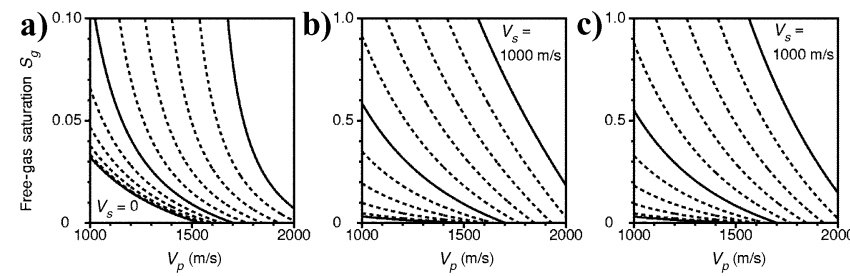

Figure 2. Free-gas saturation $S_{g}$ as a function of $v_{p}$ and $v_{s}$. (a) Homogeneous gas distribution (equation 7). (b) Patchy gas distribution (equation 8). (c) Same as (b) but approximate solution (equation 9). The $\mathrm{S}$-wave velocity is shown in steps of $100 \mathrm{~m} / \mathrm{s}(0,500$, and $1000 \mathrm{~m} / \mathrm{s}$ as solid lines). The same parameters are used as in Figure $1 \mathrm{~b}$, except for $\gamma_{\text {dry }}^{2}=2.4$ and $K_{g}=0.05 \mathrm{GPa}$. 
gas-hydrate-bearing sediments from seismic or borehole data in only a very limited number of studies, three of which are discussed here. The authors of two seismic studies determine the saturations by using the Frenkel-Gassmann equations or the Geertsma-Smit equations, with the saturation in implicit form. I investigate which measured input variables are important for the accuracy of the saturation estimate by using the partial derivatives of Appendix C. The third study is related to borehole measurements from ODP Leg 164 and is used here to compare the saturation estimate from seismic methods with saturations determined from pore water chloride anomalies and from pressure core samples.

Tinivella and Accaino (2000) analyze seismic data from the antarctic Shetland margin; the data were recorded by a multichannel streamer and an ocean-bottom seismometer (OBS). The water depth at the OBS position is $1780 \mathrm{~m}$; the bottom-simulating reflector (BSR), which is the interface between gas-hydrate- and freegas-bearing sediments, is located at $600 \mathrm{~m}$ bsf. P- and S-wave velocities were determined by traveltime inversion and AVO analysis. P-wave velocities of 2000-2300 m/s and 1200-1500 $\mathrm{m} / \mathrm{s}$ were obtained for the gas-hydrate and the free-gas zones at the BSR depth, and the S-wave velocity was determined to be 700 $-900 \mathrm{~m} / \mathrm{s}$ in both regions.

The authors use an empirical density-depth function for terrigenous sediments (Hamilton, 1976), which leads to a density of $2.1 \mathrm{~g} / \mathrm{cm}^{3}$ at the BSR depth, and they report that the density did not change significantly during the $\mathrm{P}$-wave amplitude inversion. They determined a peak value of $23 \%$ for the gas-hydrate saturation and a value of $6 \%$ for the free-gas saturation (homogeneous distribution), and they state the free-gas saturation was difficult to determine with good accuracy. I compute the gas-hydrate and free-gas saturations with equations 4,7 , and 9 and the standard deviations with the formulas of Appendix $\mathrm{C}$ by using the parameters of Table 2. This leads to $24 \% \pm 11 \%$ gas hydrate, $6 \% \pm 3 \%$ free gas for a homogeneous gas distribution, and $37 \% \pm 12 \%$ free gas for patchy gas distribution (Figure 3). These high values for the free-gas saturation are obtained for $\mathrm{P}$ - and $\mathrm{S}$-wave velocities of 1500 and $700 \mathrm{~m} / \mathrm{s}$, respectively. The gas saturation takes unrealistically high values of $30 \%$ for the homogeneous distribution and $80 \%$ for the patchy distribution for P- and S-wave velocities of 1300 and $800 \mathrm{~m} / \mathrm{s}$, which are the velocities at the OBS position.

Tinivella and Accaino (2000) mention that the free-gas saturation for the homogeneous distribution cannot be determined with good accuracy. This can be explained in the following way: The denominator of equation C- 8 is small in the limit $M \rightarrow 0$, where the function $M$ is defined by equation 5 . This is the case if the $\mathrm{P}$ - to $\mathrm{S}$-wave velocity ratio is the same for the wet and dry medium, i.e., the measured (wet) P-wave velocity is approximately 1.5 times higher than the S-wave velocity. Lodolo et al. (2002) perform a traveltime inversion on P-wave data from the same survey area but do not investigate $\mathrm{S}$-waves. They report much smaller values: $4 \%$ gas hydrate for a P-wave velocity of $2000 \mathrm{~m} / \mathrm{s}$ and $0.2 \%$ free gas for a P-wave velocity of $1750 \mathrm{~m} / \mathrm{s}$ (Figure 3 ). The gas saturation should be three times higher because the authors use an incorrect value for the gas bulk modulus. These results can be obtained from equations 4 and 7 only for smaller S-wave velocities of 600 and $400 \mathrm{~m} / \mathrm{s}$, for which experimental evidence is missing. This example demonstrates that the saturation estimates strongly depend on the S-wave velocity, which was obtained for the survey area by analyzing a single OBS station.

Andreassen et al. (2003) analyze seismic data from an ocean-

bottom cable (OBC) experiment at the Storegga slide, offshore Norway, which was performed at a geotechnical borehole where the water depth is $980 \mathrm{~m}$ and the BSR depth is $270 \mathrm{~m} \mathrm{bsf}$. The authors used seismic ray tracing and finite-difference modeling to obtain P- and S-wave velocities of 1600 and $435 \mathrm{~m} / \mathrm{s}$ in the gas hydrate layer at the BSR depth near the borehole location. A previous vertical seismic profiling experiment and the waveform inversion of seismic multichannel data had led to higher P-wave velocities around $1800 \mathrm{~m} / \mathrm{s}$ at the BSR depth (Andreassen et al., 2000). Bünz

Table 2. Elastic properties of the marine sediment at the antarctic Shetland margin at BSR depth according to Tinivella and Accaino (2000). The gas-hydrate saturation is given by $S_{h}=23.8 \% \pm 10.8 \%$, and the free-gas saturation for the homogeneous distribution is $S_{g}=5.9 \% \pm 3.2 \%$, and for the patching distribution is $S_{g}=13.9 \% \pm 36.9 \%$. The standard deviation $\Delta S$ is computed with the formulas of Appendix $\mathrm{C}$ by assuming plausible values for the standard deviations of the input parameters.

Independent variables

$v_{p} \pm \Delta v_{p}=2.3 \pm 0.05 \mathrm{~km} / \mathrm{s}$

$v_{s} \pm \Delta v_{s}=0.8 \pm 0.05 \mathrm{~km} / \mathrm{s}$

$\gamma_{\text {dry }}^{2} \pm \Delta \gamma_{\text {dry }}^{2}=2.7 \pm 0.3$

$K_{s} \pm \Delta K_{s}=37 \pm 5 \mathrm{GPa}$

$K_{w} \pm \Delta K_{w}=2.5 \pm 0.05 \mathrm{GPa}$

$\rho \pm \Delta \rho=2.1 \pm 0.05 \mathrm{~g} / \mathrm{cm}^{3}$

$\rho_{s} \pm \Delta \rho_{s}=2.65 \pm 0.05 \mathrm{~g} / \mathrm{cm}^{3}$

$\rho_{w} \pm \Delta \rho_{w}=1.03 \pm 0.005 \mathrm{~g} / \mathrm{cm}^{3}$

$\rho_{h} \pm \Delta \rho_{h}=0.93 \pm 0.02 \mathrm{~g} / \mathrm{cm}^{3}$

$v_{p} \pm \Delta v_{p}=1.5 \pm 0.05 \mathrm{~km} / \mathrm{s}$

$v_{s} \pm \Delta v_{s}=0.7 \pm 0.05 \mathrm{~km} / \mathrm{s}$

$\gamma_{\text {dry }}^{2} \pm \Delta \gamma_{\text {dry }}^{2}=2.4 \pm 0.3$

$K_{s} \pm \Delta K_{s}=37 \pm 5 \mathrm{GPa}$

$K_{w} \pm \Delta K_{w}=2.5 \pm 0.05 \mathrm{GPa}$

$\rho \pm \Delta \rho=2.1 \pm 0.05 \mathrm{~g} / \mathrm{cm}^{3}$

$\rho_{s} \pm \Delta \rho_{s}=2.65 \pm 0.05 \mathrm{~g} / \mathrm{cm}^{3}$

$\rho_{w} \pm \Delta \rho_{w}=1.03 \pm 0.005 \mathrm{~g} / \mathrm{cm}^{3}$

$\rho_{g} \pm \Delta \rho_{g}=0.2 \pm 0.05 \mathrm{~g} / \mathrm{cm}^{3}$

$K_{g} \pm \Delta K_{g}=0.07 \pm 0.01 \mathrm{GPa}$
Saturation error

$$
\begin{aligned}
& \Delta S_{h}=4.9 \% \\
& \Delta S_{h}=-4.6 \% \\
& \Delta S_{h}=-4.1 \% \\
& \Delta S_{h}=-2.2 \% \\
& \Delta S_{h}=-1.5 \% \\
& \Delta S_{h}=-5.1 \% \\
& \Delta S_{h}=4.7 \% \\
& \Delta S_{h}=0.2 \% \\
& \Delta S_{h}=0.2 \% \\
& \Delta S_{g}=-1.3(-4.7) \% \\
& \Delta S_{g}=2.2(8.1) \% \\
& \Delta S_{g}=1.3(7.1) \% \\
& \Delta S_{g}=0.1(0.3) \% \\
& \Delta S_{g}=0.1(0.3) \% \\
& \Delta S_{g}=0.6(1.3) \% \\
& \Delta S_{g}=-0.5(-1.1) \% \\
& \Delta S_{g}=0.0(-0.3) \% \\
& \Delta S_{g}=0.0 \% \\
& \Delta S_{g}=0.8(0.6) \%
\end{aligned}
$$
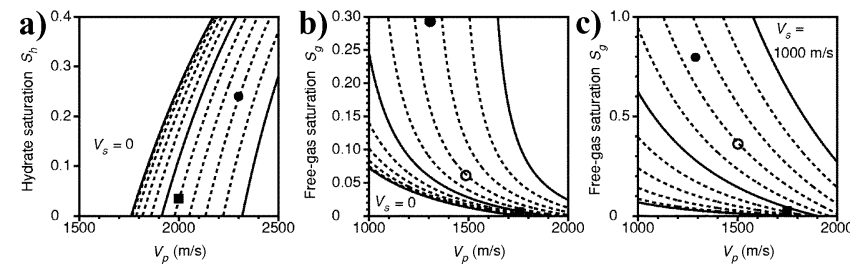

Figure 3. (a) Gas-hydrate saturation, (b) free-gas saturation (homogeneous distribution), and (c) free-gas saturation (patchy distribution) at the antarctic Shetland margin at the BSR depth computed with equations 4,7 , and 9 by using parameters from Tinivella and Accaino (2000) (circles) and from Lodolo et al. (2002) (squares). The curves are computed with the parameters of Table 2 for $\mathrm{S}$-wave velocities between 0 and $1000 \mathrm{~m} / \mathrm{s}$ in $100-\mathrm{m} / \mathrm{s}$ increments. 
and Mienert (2004) apply standard seismic processing to the same $\mathrm{OBC}$ data set and obtain laterally varying $\mathrm{P}$-wave velocities of $1760-1870 \mathrm{~m} / \mathrm{s}$ at the BSR depth, but they do not investigate S-waves.

Using equation 4 and the parameters of Table 3, I obtain 12\% $\pm 9 \%$ gas hydrate. The large inaccuracy of this result is caused by uncertainties in P- and S-wave velocities and bulk density. Andreassen et al. (2003) estimate 1\%-1.5\% gas hydrate, but Figure 4 shows no gas hydrate in the sediment for P-wave velocities of $1600 \mathrm{~m} / \mathrm{s}$ unless the $\mathrm{S}$-wave velocity is smaller than $200 \mathrm{~m} / \mathrm{s}$. This is not the case, since the authors report that the S-wave velocities take values of $300-500 \mathrm{~m} / \mathrm{s}$ in the target area. Bünz and Mienert (2004) obtain $5 \% \pm 5 \%$ gas hydrate, which I obtain for a P-wave velocity of $1750 \mathrm{~m} / \mathrm{s}$. The P-wave velocities of $1300-1400 \mathrm{~m} / \mathrm{s}$ in the free-gas layer lead to $1.2 \% \pm 0.6 \%$ free gas for the homogeneous distribution, which confirms the result of Bünz and Mienert (2004). Zillmer et al. (2005b) accidentally use a lower S-wave velocity of 375 instead of $435 \mathrm{~m} / \mathrm{s}$ and obtain $0.8 \%$ free gas. Andreassen et al. (2003) report $0.45 \%$ free gas, but they use a value that is too large for the gas bulk modulus, so the actual result for the saturation, which depends linearly on $K_{g}$, is even smaller and cannot be confirmed here.

The gas-hydrate and free-gas saturations as functions of all input variables are shown in Figure 5. The seismic P- and S-wave veloci-

Table 3. Elastic properties of the marine sediment at the Storegga slide, offshore Norway, at BSR depth according to Andreassen et al. (2000, 2003) and Bünz and Mienert (2004). The gas-hydrate saturation is given by $S_{h}=12.1 \% \pm 8.6 \%$, and the free-gas saturation for the homogeneous distribution is $S_{g}=1.2 \% \pm 0.6 \%$ and for the patchy distribution is $S_{g}$ $=13.9 \% \pm 5.6 \%$ The standard deviation $\Delta S$ is computed with the formulas of Appendix $C$ by assuming plausible values for the standard deviations of the input parameters.

Independent variables

\begin{tabular}{ll}
\hline$v_{p} \pm \Delta v_{p}=1.8 \pm 0.05 \mathrm{~km} / \mathrm{s}$ & $\Delta S_{h}=5.8 \%$ \\
$v_{s} \pm \Delta v_{s}=0.435 \pm 0.05 \mathrm{~km} / \mathrm{s}$ & $\Delta S_{h}=-3.8 \%$ \\
$\gamma_{\text {dry }}^{2} \pm \Delta \gamma_{\text {dry }}^{2}=2.7 \pm 0.3$ & $\Delta S_{h}=-1.8 \%$ \\
$K_{s} \pm \Delta K_{s}=40 \pm 5 \mathrm{GPa}$ & $\Delta S_{h}=-1.0 \%$ \\
$K_{w} \pm \Delta K_{w}=2.4 \pm 0.05 \mathrm{GPa}$ & $\Delta S_{h}=-1.8 \%$ \\
$\rho \pm \Delta \rho=1.85 \pm 0.05 \mathrm{~g} / \mathrm{cm}^{3}$ & $\Delta S_{h}=-3.1 \%$ \\
$\rho_{s} \pm \Delta \rho_{s}=2.65 \pm 0.05 \mathrm{~g} / \mathrm{cm}^{3}$ & $\Delta S_{h}=2.9 \%$ \\
$\rho_{w} \pm \Delta \rho_{w}=1.03 \pm 0.005 \mathrm{~g} / \mathrm{cm}^{3}$ & $\Delta S_{h}=0.3 \%$ \\
$\rho_{h} \pm \Delta \rho_{h}=0.93 \pm 0.02 \mathrm{~g} / \mathrm{cm}^{3}$ & $\Delta S_{h}=0.1 \%$ \\
$v_{p} \pm \Delta v_{p}=1.3 \pm 0.05 \mathrm{~km} / \mathrm{s}$ & $\Delta S_{g}=-0.3(-2.4) \%$ \\
$v_{s} \pm \Delta v_{s}=0.435 \pm 0.05 \mathrm{~km} / \mathrm{s}$ & $\Delta S_{g}=0.2(4.4) \%$ \\
$\gamma_{\text {dry }}^{2} \pm \Delta \gamma_{\text {dry }}^{2}=2.4 \pm 0.3$ & $\Delta S_{g}=0.1(2.4) \%$ \\
$K_{s} \pm \Delta K_{s}=40 \pm 5 \mathrm{GPa}$ & $\Delta S_{g}=0.0(0.1) \%$ \\
$K_{w} \pm \Delta K_{w}=2.4 \pm 0.05 \mathrm{GPa}$ & $\Delta S_{g}=0.0(0.3) \%$ \\
$\rho \pm \Delta \rho=1.85 \pm 0.05 \mathrm{~g} / \mathrm{cm}^{3}$ & $\Delta S_{g}=0.1(0.5) \%$ \\
$\rho_{s} \pm \Delta \rho_{s}=2.65 \pm 0.05 \mathrm{~g} / \mathrm{cm}^{3}$ & $\Delta S_{g}=-0.1(-0.4) \%$ \\
$\rho_{w} \pm \Delta \rho_{w}=1.03 \pm 0.005 \mathrm{~g} / \mathrm{cm}^{3}$ & $\Delta S_{g}=0.0(-0.1) \%$ \\
$\rho_{g} \pm \Delta \rho_{g}=0.12 \pm 0.05 \mathrm{~g} / \mathrm{cm}^{3}$ & $\Delta S_{g}=0.0 \%$ \\
$K_{g} \pm \Delta K_{g}=0.03 \pm 0.01 \mathrm{GPa}$ & $\Delta S_{g}=0.4(0.4) \%$
\end{tabular}

ties are important for the gas-hydrate and free-gas saturation. The gas-hydrate saturation also requires a precise value for the bulk density. The pore water bulk modulus should be computed carefully as a function of pressure (Dvorkin et al., 1999). The average grain density of the material should be known, while the average bulk modulus is only important for values smaller than $20 \mathrm{GPa}-$ that is, for sediments which contain large amounts of gas hydrate.
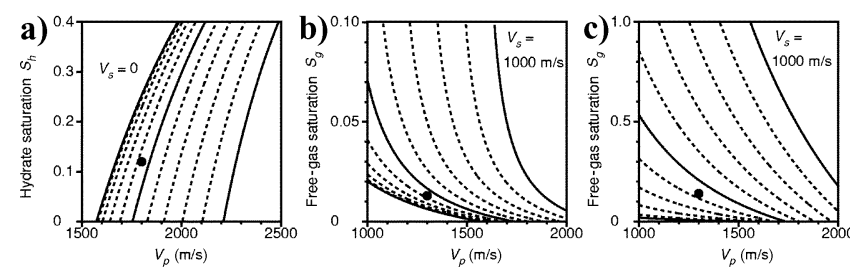

Figure 4. (a) Gas-hydrate saturation, (b) free-gas saturation (homogeneous distribution), and (c) free-gas saturation (patchy distribution), at the Storegga slide, offshore Norway, computed with equations 4, 7, and 9 by using parameters of Andreassen et al. (2000, 2003) and Bünz and Mienert (2004). The curves are computed with the parameters of Table 3 for S-wave velocities between 0 and $1000 \mathrm{~m} / \mathrm{s}$ in $100-\mathrm{m} / \mathrm{s}$ increments.
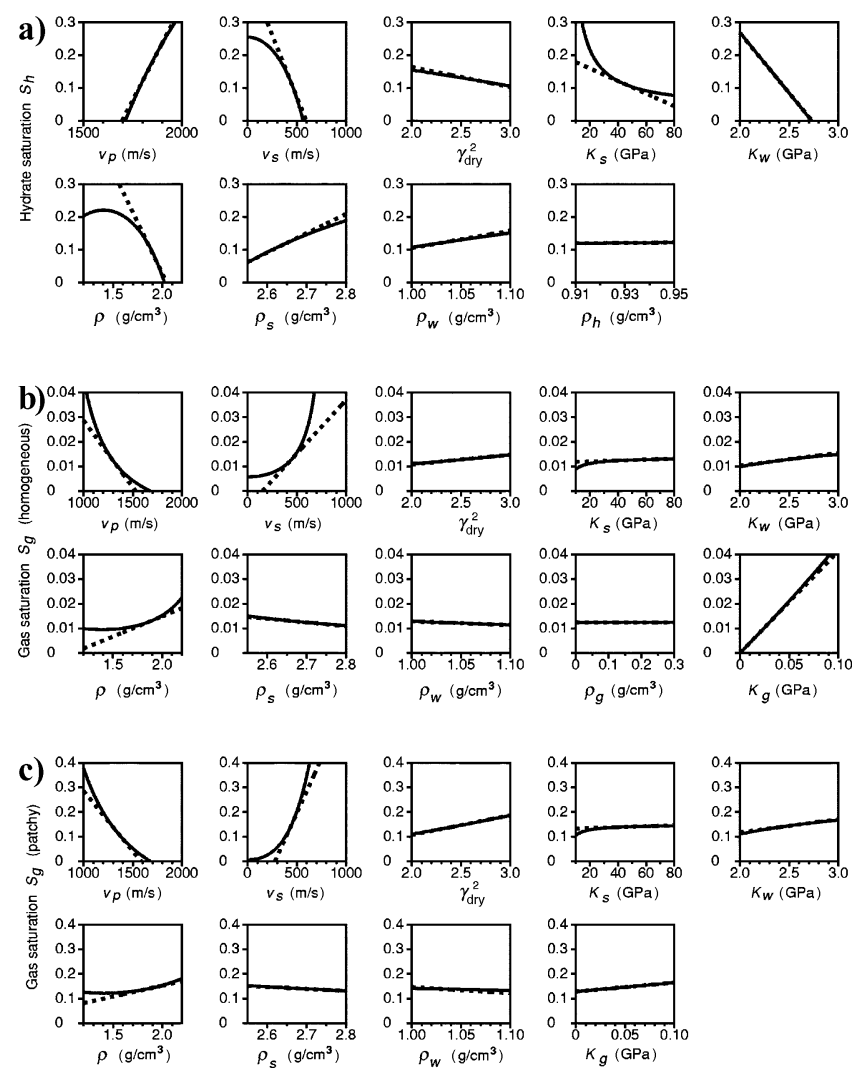

Figure 5. (a) Gas-hydrate saturation, (b) free-gas saturation (homogeneous distribution), and (c) free-gas saturation (patchy distribution) at the Storegga slide as functions of the input variables. The saturations (solid lines) are computed with equations 4,7 , and 9, and the linearized solutions (dotted lines) are computed with formulas in Appendix C. The linearized curve is used to compute the standard deviation of the saturation based on assumptions about the standard deviations of the independent variables (see Table 3). 
The goal of ODP Leg 164 was to study gas-hydrate occurrences at Blake Ridge, offshore South Carolina (Paull et al., 1996, 2000). Drillholes at three sites penetrated the base of the gas-hydrate stability zone. The water depth at site 995 is $2780 \mathrm{~m}$, and the BSR is located at about $450 \mathrm{~m}$ bsf. Guerin et al. (1999) derive P- and $\mathrm{S}$-wave velocity-depth profiles from dipole sonic-log measurements, which they use together with the bulk densities and other

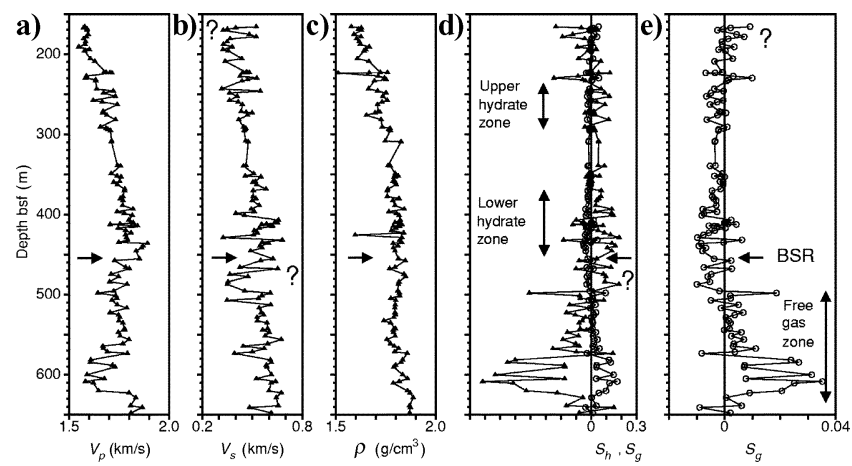

Figure 6. Gas-hydrate and free-gas saturations at ODP Leg 164, site 995 (Blake Ridge), as a function of depth below the sea floor. (a) P-wave sonic log (Paull et al., 1996). (b) S-wave sonic log (after Guerin et al., 1999). (c) Bulk density from cores (Paull et al., 1996). (d) Gas-hydrate saturation computed with equation 4 and free-gas saturation of patchy distribution computed with equation 9. (e) Free-gas saturation of a homogeneous distribution computed with equation 7. The following values are used: $K_{s}=30 \mathrm{GPa}, \rho_{s}$ $=2.7 \mathrm{~g} / \mathrm{cm}^{3}, \gamma_{\mathrm{dry}}^{2}=2.7$ for the gas-hydrate saturation, and $\gamma_{\mathrm{dry}}^{2}$ $=2.4$ for the free-gas saturation. Between 150 and $650 \mathrm{~m}$ bsf, the pressure increases from 30 to $39 \mathrm{MPa}$ and the temperature increases from $8^{\circ}$ to $25^{\circ} \mathrm{C}$; the salinity of the pore water is $\sim 32 \mathrm{ppt}$ (Paull et al., 1996). The density $\rho_{w}$ of the pore water is $\sim 1.035$ $\mathrm{g} / \mathrm{cm}^{3}$, and the bulk modulus $K_{w}$ increases from 2.41 to 2.62 $\mathrm{GPa}$; the density $\rho_{g}$ of the free gas is $0.24-0.26 \mathrm{~g} / \mathrm{cm}^{3}$, and the bulk modulus $K_{g}$ increases from 0.10 to $0.14 \mathrm{GPa}$ (Batzle and Wang, 1992). Question marks indicate doubts about the S-wave velocities and the corresponding gas-hydrate and free-gas saturations.

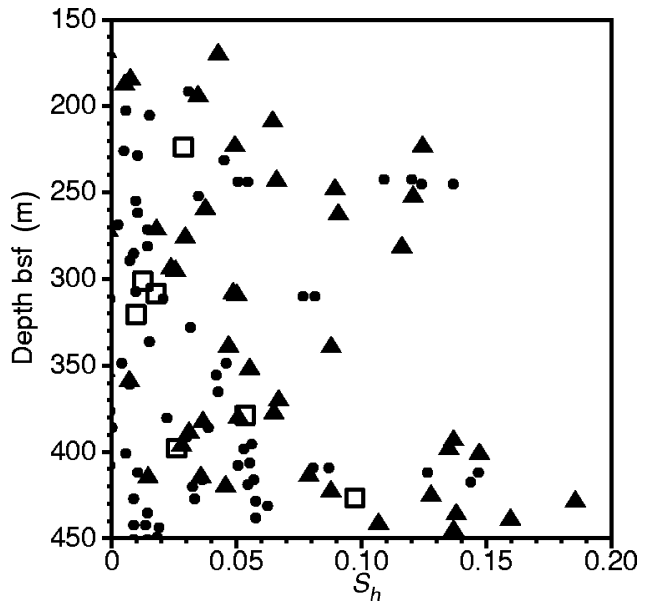

Figure 7. Gas-hydrate saturation (triangles) at ODP site 995 computed from equation 4 for the depth interval between 150 and $450 \mathrm{~m}$ bsf as a zoom of Figure 6d. Gas-hydrate saturation is estimated from interstitial pore water chloride anomalies (dots) (after Collett and Ladd, 2000) and from pressure core samples (squares) (after Guerin et al., 1999). $\log$ data to investigate gas-hydrate saturation. I use densities obtained from borehole cores, which are more reliable than log densities (Collett and Ladd, 2000).

The bulk density as a function of depth and corresponding Pand S-wave velocities from the logs are shown in Figure 6 together with the gas-hydrate and free-gas saturations. The gas-hydrate saturation takes peak values around $12 \%$ in the depth interval between 220 and $280 \mathrm{~m}$ bsf and $14 \%$ at 390-450 m depth bsf. These results coincide qualitatively with estimates from pore water chloride anomalies (Collett and Ladd, 2000) and from pressure core samples (Guerin et al., 1999) (Figure 7). The results are similar to those of Tinivella and Lodolo (2000), who use the Geertsma-Smit equation, and to Jacobsen et al. (2000), who use the SCA/DEM method. The maximum amount of free gas is located at $600 \mathrm{~m} \mathrm{bsf}$, with values of $3.5 \%$ for the homogeneous and $17 \%$ for the patchy distribution. The S-wave velocities in the depth interval between 160 and $200 \mathrm{~m}$ bsf seem to be too high and erroneously indicate the presence of free gas. According to the function in Figure 6d, gas hydrate occurs below the BSR at $450-520 \mathrm{~m}$ bsf. This result is caused by a strong decrease in S-wave velocity, which is accompanied by only a smaller decrease in P-wave velocity and a constant bulk density.

Guerin et al. (1999) construct a model where gas hydrate and free gas are both present and the effects on the elastic properties are compensated for. The gas-hydrate saturation can then be computed as a function of the free-gas saturation, which in turn must be guessed. The caliper log shows large borehole diameters in the depth interval between 470 and $510 \mathrm{~m}$ that are close to the 46.9$\mathrm{cm}$ maximum range of the caliper arm (Collett and Ladd, 2000). I speculate that the $\mathrm{S}$-wave velocities are too low and that there is no gas hydrate but rather a small amount of free gas in this depth interval.

\section{CONCLUSIONS}

Simple equations for the gas-hydrate and free-gas saturations have been derived in explicit form based on the Frenkel-Gassmann equations. The obtained gas-hydrate-saturation estimates for the borehole data of ODP Leg 164 coincide qualitatively with results of measurements from a pressure core sampler and with estimates from pore water chloride anomalies. The saturation also coincides with results of a multiphase effective medium (SCA/DEM) method. These results indicate that the equations presented here can be used to determine gas-hydrate saturation in porous media for large wavelengths. A sensitivity analysis reveals that the most important parameters for determining saturation are the two seismic velocities and, in the case of gas hydrate, the bulk density. The pore water bulk modulus should be computed carefully as a function of pressure for accurately determining gas-hydrate saturation. The freegas saturation for the homogeneous distribution is proportional to the gas bulk modulus, which should be determined carefully as a function of pressure - a point that is often neglected. In the case of patchy gas distribution, the precise value of the gas bulk modulus is not important if it is much smaller than the bulk modulus of pore water, which is the case for methane gas. The free-gas saturation for the homogeneous model is unstable with respect to inaccuracies in the velocities if the ratio of P-to $\mathrm{S}$-wave velocity is equal for the wet and dry medium, which means that the measured (wet) $\mathrm{P}$-wave velocity is about 1.5 times higher than the $\mathrm{S}$-wave velocity. 


\section{ACKNOWLEDGMENTS}

This study was supported by the European Union (CRIMEA project, EVK2-CT-2002-00162). The author thanks the associate editor and an anonymous reviewer for their comments on the manuscript.

\section{APPENDIX A \\ DERIVATION OF THE EQUATIONS FOR GAS-HYDRATE AND FREE-GAS SATURATION}

I first discuss the case where gas hydrate is present in the sediment. Gas hydrate as a solid substance reduces the volume, which is occupied by the pore fluid. This is expressed by the equation

$$
S_{h}=1-\frac{\Omega}{\phi}
$$

where $S_{h}$ is the gas-hydrate saturation of the pore space and where $\phi$ and $\Omega$ are porosity and reduced porosity, respectively. The bulk density $\rho$ of the gas-hydrate-bearing sediment is given by the volumetric average of the densities of solid $\rho_{s}$, pore water $\rho_{w}$, and gas hydrate $\rho_{h}$ :

$$
\rho=(1-\phi) \rho_{s}+\Omega \rho_{w}+(\phi-\Omega) \rho_{h} .
$$

The Frenkel-Gassmann equations connect the shear moduli of the wet and dry media $\mu$ and $\mu_{\text {dry }}$,

$$
\mu=\mu_{\mathrm{dry}},
$$

and the bulk moduli $K$ and $K_{\mathrm{dry}}$,

$$
\frac{\sigma^{2}}{K-K_{\mathrm{dry}}}=\frac{\Omega}{K_{w}}+\frac{\sigma-\Omega}{K_{s}} .
$$

Here, $K_{s}$ and $K_{w}$ are the bulk moduli of solid material and of pore water and $\sigma$ is given by

$$
\sigma=1-\frac{K_{\mathrm{dry}}}{K_{s}}
$$

Note that the reduced porosity is used in equation A-4. Bulk and shear moduli are connected to the $\mathrm{P}$ - and $\mathrm{S}$-wave velocities $v_{p}$ and $v_{s}$ by

$$
K=\rho\left(v_{p}^{2}-\frac{4}{3} v_{s}^{2}\right)
$$

and

$$
\mu=\rho v_{s}^{2} .
$$

The ratio $\gamma_{\text {dry }}$ of the $\mathrm{P}$ - and $\mathrm{S}$-wave velocities of the dry material is given by

$$
\gamma_{\text {dry }}^{2}=\left(\frac{v_{p, \text { dry }}}{v_{s, \text { dry }}}\right)^{2}=\frac{K_{\text {dry }}}{\mu_{\text {dry }}}+\frac{4}{3}=\frac{2-2 \nu_{\text {dry }}}{1-2 \nu_{\text {dry }}}
$$

where $\nu_{\text {dry }}$ is Poisson's ratio of the dry material. From equations A-3, A-7, and A-8 follows

$$
K_{\text {dry }}=\left(\gamma_{\text {dry }}^{2}-\frac{4}{3}\right) \rho v_{s}^{2}
$$

The gas-hydrate saturation $S_{h}$ (equation 4) is obtained from equation A-1 by eliminating the variables $\phi$ and $\Omega$ using equations A-2 and A-4. The final form of equation 4 is obtained with equations A-6 and A-9.

Next, I discuss the case of a sediment where free gas and water are present in the pore space. Following Helgerud et al. (1999), I distinguish two models: a homogeneous distribution, i.e., the same amount of free gas and water in each pore, and a patchy distribution, which means that many pores are fully water saturated while others are fully gas saturated. In the case of the model with a homogeneous distribution of free gas in the pore space, the saturation $S_{g}$ is obtained from three equations. The density equation is

$$
\rho=(1-\phi) \rho_{s}+\phi\left(1-S_{g}\right) \rho_{w}+\phi S_{g} \rho_{g},
$$

where $\rho_{g}$ is the density of the gas. The Frenkel-Gassmann equation is

$$
\frac{\sigma^{2}}{K-K_{\mathrm{dry}}}=\frac{\phi}{K_{f}}+\frac{\sigma-\phi}{K_{s}}
$$

The equation for the bulk modulus of the pore fluid $K_{f}$, which is computed as the Reuss average of water and gas bulk moduli, is

$$
\frac{1}{K_{f}}=\frac{S_{g}}{K_{g}}+\frac{1-S_{g}}{K_{w}}
$$

Here, $K_{g}$ is the adiabatic bulk modulus of the gas. The final form of equation 7 is obtained by using equations A- 6 and A-9.

In a second model, patches of fully gas- and fully water-saturated sediment occupy neighboring regions on a length scale much smaller than the wavelength. According to equation A-3, the pore fluid (gas or water) does not affect the shear modulus. In this case the inverse P-wave moduli can be averaged to obtain the average P-wave modulus (Hill, 1963):

$$
\begin{aligned}
\left(K+\frac{4}{3} \rho v_{s}^{2}\right)^{-1}= & S_{g}\left[K_{\mathrm{dry}}+\sigma^{2}\left(\frac{\phi}{K_{g}}+\frac{\sigma-\phi}{K_{s}}\right)^{-1}\right. \\
& \left.+\frac{4}{3} \rho v_{s}^{2}\right]^{-1}+\left(1-S_{g}\right)\left[K_{\mathrm{dry}}+\sigma^{2}\left(\frac{\phi}{K_{w}}\right.\right. \\
& \left.\left.+\frac{\sigma-\phi}{K_{s}}\right)^{-1}+\frac{4}{3} \rho v_{s}^{2}\right]^{-1}
\end{aligned}
$$

Equations A-6 and A-11 are used with $K_{f}=K_{g}$ and $K_{f}=K_{w}$, respectively. The quadratic equation 8 for the gas saturation follows from equations A-9, A-10, and A-13. The direct solution for $S_{g}$ 
(equation 9) is obtained by neglecting the terms proportional to $S_{g}$ in equation A-10. The approximation is valid if

$$
S_{g} \ll \frac{\rho_{s}-\rho_{w}}{\rho_{w}-\rho_{g}} .
$$

This condition is fulfilled if $\rho_{s} \gg 2 \rho_{w}$; thus, equation 9 is probably always a good approximation for the free-gas saturation of marine sediments.

\section{APPENDIX B}

\section{THE RATIO OF P-AND S-WAVE VELOCITY FOR AN AGGREGATE OF ELASTIC SPHERES AT HIGH POROSITY}

The dry sediment frame under hydrostatic pressure is often represented by a packing of elastic spheres. Wang and Nur (1992) and Mavko et al. (1998) present short reviews of theories that treat the contact of elastic spheres under pressure. Some results of different contact theories for the ratio between the bulk and shear modulus are summarized in this appendix. I assume that the spheres have Poisson ratios $\nu_{s}$ between 0 and 0.5 , which is the range for common minerals (Mavko et al., 1998).

A dense random packing of spheres has a critical porosity of $\phi_{c} \approx 0.36$ (Dvorkin et al., 1999). For this case, the Hertz-Mindlin contact theory (Mindlin, 1949) leads to the following ratio:

$$
\frac{K_{c}}{\mu_{c}}=\frac{5\left(2-\nu_{s}\right)}{3\left(5-4 \nu_{s}\right)}
$$

with the notation $K_{c}=K_{\text {dry }}\left(\phi=\phi_{c}\right)$ and $\mu_{c}=\mu_{\text {dry }}\left(\phi=\phi_{c}\right)$. For Poisson ratios between 0 and 0.5 , one obtains

$$
\frac{2}{3} \leq \frac{K_{c}}{\mu_{c}} \leq \frac{5}{6}
$$

The interval $\phi_{c} \leq \phi \leq 1$ is called the suspension domain. Dvorkin et al. (1999) and Helgerud et al. (1999) compute the dry elastic moduli in the suspension domain with a modified HashinShtrikman upper bound. This can be written as

$$
K_{\mathrm{dry}}\left(\phi_{c} \leq \phi \leq 1\right)=(1-\phi)\left[\frac{1-\phi_{c}}{K_{c}}+\frac{\phi-\phi_{c}}{\frac{4}{3} \mu_{c}}\right]^{-1}
$$

and

$\mu_{\mathrm{dry}}\left(\phi_{c} \leq \phi \leq 1\right)=(1-\phi)\left[\frac{1-\phi_{c}}{\mu_{c}}+\frac{\phi-\phi_{c}}{Z}\right]^{-1}$, where

$$
Z=\frac{9 K_{c}+8 \mu_{c}}{K_{c}+2 \mu_{c}} \frac{\mu_{c}}{6} .
$$

One obtains

$$
\frac{K_{\mathrm{dry}}}{\mu_{\mathrm{dry}}}\left(\phi_{c} \leq \phi<1\right)=\frac{4}{3} \frac{K_{c}}{Z} \frac{\left(1-\phi_{c}\right) Z+\left(\phi-\phi_{c}\right) \mu_{c}}{\left(1-\phi_{c}\right) \frac{4}{3} \mu_{c}+\left(\phi-\phi_{c}\right) K_{c}} .
$$

If $K_{c} / \mu_{c}<4 / 3$, then $K_{\text {dry }} / \mu_{\text {dry }}$ is a monotonic increasing function of $\phi$ in the suspension domain with maximum

$$
\frac{K_{\text {dry }}}{\mu_{\text {dry }}}(\phi \rightarrow 1) \rightarrow \frac{20 \frac{K_{c}}{\mu_{c}}}{8+9 \frac{K_{c}}{\mu_{c}}},
$$

which follows from equations B-5 and B-6. Using equation B-1, it follows that

$$
\frac{K_{\mathrm{dry}}}{\mu_{\mathrm{dry}}}(\phi \rightarrow 1) \rightarrow \frac{100}{3} \frac{2-\nu_{s}}{70-47 \nu_{s}}
$$

For Poisson ratios between 0 and 0.5 ,

$$
\frac{20}{21} \leq \frac{K_{\text {dry }}}{\mu_{\text {dry }}}(\phi \rightarrow 1) \leq \frac{100}{93}
$$

Some results for other sphere packings and contact conditions are summarized in the following.

The contact theory of Walton (1987) for rough spheres leads again to equation B-1, whereas for smooth spheres Walton obtains

$$
\frac{K_{\text {dry }}}{\mu_{\text {dry }}}=\frac{5}{3},
$$

which is also the maximum ratio for the cementation model of Dvorkin et al. (1994)

Digby (1981) investigates spheres with adhesion that are already bonded before a hydrostatic pressure is applied. This model leads to

$$
\frac{K_{\mathrm{dry}}}{\mu_{\mathrm{dry}}}=\frac{5}{3} \frac{2-\nu_{s}}{2-\nu_{s}+3\left(1-\nu_{s}\right) \frac{b}{a}},
$$

where $b$ is the initial radius and $a$ is the final contact radius. The relation $a \geq b$ leads to

$$
\frac{5}{3} \frac{2-\nu_{s}}{5-4 \nu_{s}} \leq \frac{K_{\text {dry }}}{\mu_{\text {dry }}} \leq \frac{5}{3} .
$$


The ratio of bulk and shear modulus of a sphere packing is

$$
\frac{2}{3} \leq \frac{K_{\mathrm{dry}}}{\mu_{\mathrm{dry}}} \leq \frac{5}{3}
$$

The square of the ratio of $\mathrm{P}$ - and S-wave velocity is

$$
2 \leq \gamma_{\text {dry }}^{2} \leq 3
$$

These results are valid for the suspension domain according to the method of Dvorkin et al. (1999) for Poisson ratios of spheres between 0 and 0.5 . They are also valid for some other contact theories.

\section{APPENDIX C}

\section{ERROR OF GAS-HYDRATE AND FREE-GAS SATURATIONS}

The variance of the saturation $S$ is computed by

$$
\operatorname{var}(S)=\sum_{i=1}^{N}\left(\frac{\partial S}{\partial x_{i}}\right)^{2} \operatorname{var}\left(x_{i}\right)
$$

where $x_{i}$ are independent variables. I linearize $S$ as

$$
S=S_{0}+\Delta S \approx S_{0}+\left.\frac{\partial S}{\partial v_{p}}\right|_{0} \Delta v_{p}+\ldots,
$$

where the ellipses indicate terms with other independent variables.

Using $K_{\text {dry }} \ll K_{s}$ in equation 4 , it follows that $\sigma \approx 1$ and

$$
\frac{\partial S_{h}}{\partial K_{s}} \approx \frac{-\left(\rho_{s}-\rho\right)\left(\rho_{s}-\rho_{h}\right)\left(\frac{1}{K_{w}}-\frac{1}{M}\right)}{K_{s}^{2}\left[\left(\rho_{s}-\rho\right)\left(\frac{1}{K_{w}}-\frac{1}{K_{s}}\right)+\left(\rho_{w}-\rho_{h}\right)\left(\frac{1}{M}-\frac{1}{K_{s}}\right)\right]^{2}} .
$$

The second term in the denominator can be neglected because water and gas hydrate have similar densities, $\rho_{w} \approx \rho_{h}$, which leads to

$$
\frac{\partial S_{h}}{\partial K_{s}} \approx \frac{-\left(\rho_{s}-\rho_{h}\right)\left(\frac{1}{K_{w}}-\frac{1}{M}\right)}{K_{s}^{2}\left(\rho_{s}-\rho\right)\left(\frac{1}{K_{w}}-\frac{1}{K_{s}}\right)^{2}}
$$

I neglect all terms proportional to $K_{s}^{-1}$ in equation 4 and calculate the partial derivatives with respect to the other independent variables. Using again $\rho_{w} \approx \rho_{h}$, it follows that

$$
\begin{aligned}
\Delta S_{h} \approx & \left(1-S_{h}\right)\left\{\frac{2 \rho v_{p}}{M} \Delta v_{p}-\frac{2 \rho \gamma_{\mathrm{dry}}^{2} v_{s}}{M} \Delta v_{s}+\frac{\rho_{s}-2 \rho}{\rho_{s}-\rho} \frac{\Delta \rho}{\rho}\right. \\
& -\frac{\rho v_{s}^{2}}{M} \Delta \gamma_{\mathrm{dry}}^{2}+\frac{\rho-\rho_{h}}{\rho_{s}-\rho} \frac{\Delta \rho_{s}}{\rho_{s}-\rho_{h}}+\frac{K_{w}}{M} \frac{\Delta \rho_{w}}{\rho_{s}-\rho} \\
& \left.+S_{h} \frac{\Delta \rho_{h}}{\rho_{s}-\rho_{h}}-\frac{\Delta K_{w}}{K_{w}}\right\}-\frac{\rho_{s}-\rho_{h}}{\rho_{s}-\rho}\left(\frac{1}{K_{w}}-\frac{1}{M}\right)\left(\frac{1}{K_{w}}\right. \\
& \left.-\frac{1}{K_{s}}\right)^{-2} \frac{\Delta K_{s}}{K_{s}^{2}} .
\end{aligned}
$$

Next, I calculate the partial derivatives of the free-gas saturation for a homogeneous distribution of gas and water. Using $K_{\text {dry }} \ll K_{s}$, it follows from equation 7 that

$\frac{\partial S_{g}}{\partial K_{s}}$

$$
\approx \frac{\left(\rho_{s}-\rho\right)\left[\left(\rho-\rho_{w}\right)\left(\frac{1}{K_{g}}-\frac{1}{K_{w}}\right)-\left(\rho_{w}-\rho_{g}\right)\left(\frac{1}{K_{w}}-\frac{1}{M}\right)\right]}{K_{s}^{2}\left[\left(\rho_{s}-\rho\right)\left(\frac{1}{K_{g}}-\frac{1}{K_{w}}\right)-\left(\rho_{w}-\rho_{g}\right)\left(\frac{1}{M}-\frac{1}{K_{s}}\right)\right]^{2}} .
$$

Usually the bulk modulus of gas is small compared to the other moduli, $K_{g} \ll K_{w}, M, K_{s}$, so for $\rho_{w} \ll \rho \ll \rho_{s}$ it follows that

$$
\frac{\partial S_{g}}{\partial K_{s}} \approx \frac{\rho-\rho_{w}}{\rho_{s}-\rho} \frac{K_{g}}{K_{s}^{2}} .
$$

For computing the other partial derivatives, I neglect terms proportional to $K_{s}^{-1}$ in equation 7 . By again using $K_{g} \ll K_{w}, M$,

$$
\begin{aligned}
\Delta S_{g} \approx & \frac{K_{g}}{M}\left\{\frac { \rho _ { s } - \rho _ { w } } { \rho _ { s } - \rho } \left[\frac{-2 \rho v_{p}}{M} \Delta v_{p}+\frac{2 \rho \gamma_{\mathrm{dry}}^{2} v_{s}}{M} \Delta v_{s}\right.\right. \\
& \left.+\left(\frac{1}{\rho_{s}-\rho}-\frac{1}{\rho}\right) \Delta \rho+\frac{\rho v_{s}^{2}}{M} \Delta \gamma_{\mathrm{dry}}^{2}\right]-\frac{\Delta \rho_{w}}{\rho_{s}-\rho} \\
& \left.-S_{g} \frac{\Delta \rho_{g}}{\rho_{s}-\rho}-\frac{\rho-\rho_{w}}{\left(\rho_{s}-\rho\right)^{2}} \Delta \rho_{s}\right\}+\frac{K_{g}}{K_{w}} \frac{\Delta K_{w}}{K_{w}} \\
& +S_{g} \frac{\Delta K_{g}}{K_{g}}+\frac{\rho-\rho_{w}}{\rho_{s}-\rho} \frac{K_{g}}{K_{s}} \frac{\Delta K_{s}}{K_{s}}
\end{aligned}
$$

The standard deviation of the free-gas saturation in the case of a patchy distribution of gas and water can be calculated with the help of the following partial derivatives. With $K_{\text {dry }} \ll K_{s}$ it follows from equation 9 that

$$
\begin{aligned}
\frac{\partial S_{g}}{\partial K_{s}} \approx & \frac{S_{g}}{K_{s}^{2}}\left(\rho-\rho_{w}\right)\left[\left(\frac{\rho_{s}-\rho_{w}}{M}-\frac{\rho_{s}-\rho}{K_{w}}-\frac{\rho-\rho_{w}}{K_{s}}\right)^{-1}\right. \\
& \left.-\left(\frac{\rho_{s}-\rho_{w}}{\gamma_{\text {dry }}^{2} \rho v_{s}^{2}}+\frac{\rho_{s}-\rho}{K_{g}}+\frac{\rho-\rho_{w}}{K_{s}}\right)^{-1}\right],
\end{aligned}
$$


Neglecting terms proportional to $K_{s}^{-1}$ in equation 9 and differentiating this equation leads to

$$
\frac{\partial S_{g}}{\partial v_{p}} \approx \frac{-2 S_{g}}{v_{p}} \frac{\gamma_{\mathrm{dry}}^{2} \rho v_{s}^{2}\left(\rho_{s}-\rho\right)+K_{w}\left(\rho_{s}-\rho_{w}\right)}{K_{w}\left(\rho_{s}-\rho_{w}\right)-M\left(\rho_{s}-\rho\right)},
$$

$$
\begin{aligned}
\frac{\partial S_{g}}{\partial v_{s}} & \\
& \approx \frac{2 \gamma_{\mathrm{dry}}^{2} v_{s}\left[\left(K_{w}+K_{g}\right)\left(\rho_{s}-\rho_{w}\right)-\left(M-\gamma_{\mathrm{dry}}^{2} \rho v_{s}^{2}\right)\left(\rho_{s}-\rho\right)\right]}{v_{p}^{2}\left(K_{w}-K_{g}\right)\left(\rho_{s}-\rho_{w}\right)},
\end{aligned}
$$

$$
\begin{aligned}
& \frac{\partial S_{g}}{\partial \gamma_{\mathrm{dry}}^{2}} \\
& \quad \approx\left(\frac{v_{s}}{v_{p}}\right)^{2} \frac{\left(K_{w}+K_{g}\right)\left(\rho_{s}-\rho_{w}\right)-\left(M-\gamma_{\mathrm{dry}}^{2} \rho v_{s}^{2}\right)\left(\rho_{s}-\rho\right)}{\left(K_{w}-K_{g}\right)\left(\rho_{s}-\rho_{w}\right)},
\end{aligned}
$$

$$
\frac{\partial S_{g}}{\partial K_{w}} \approx \frac{S_{g}}{K_{w}-K_{g}} \frac{M\left(\rho_{s}-\rho\right)-K_{g}\left(\rho_{s}-\rho_{w}\right)}{K_{w}\left(\rho_{s}-\rho_{w}\right)-M\left(\rho_{s}-\rho\right)},
$$

$$
\frac{\partial S_{g}}{\partial K_{g}} \approx \frac{S_{g}}{K_{w}-K_{g}} \frac{K_{w}\left(\rho_{s}-\rho_{w}\right)+\gamma_{\mathrm{dry}}^{2} \rho v_{s}^{2}\left(\rho_{s}-\rho\right)}{K_{g}\left(\rho_{s}-\rho_{w}\right)+\gamma_{\mathrm{dry}}^{2} \rho v_{s}^{2}\left(\rho_{s}-\rho\right)}
$$

$$
\begin{aligned}
\frac{\partial S_{g}}{\partial \rho} \approx & S_{g} \frac{\rho_{s}-2 \rho}{\rho}\left[\frac{M}{M\left(\rho_{s}-\rho\right)-K_{w}\left(\rho_{s}-\rho_{w}\right)}-\frac{1}{\rho_{s}-\rho}\right. \\
& \left.+\frac{\gamma_{\mathrm{dry}}^{2} \rho v_{s}^{2}}{K_{g}\left(\rho_{s}-\rho_{w}\right)+\gamma_{\mathrm{dry}}^{2} \rho v_{s}^{2}\left(\rho_{s}-\rho\right)}\right],
\end{aligned}
$$

$$
\begin{aligned}
\frac{\partial S_{g}}{\partial \rho_{s}} \approx & -S_{g}\left[\frac{M-K_{w}}{K_{w}\left(\rho_{s}-\rho_{w}\right)-M\left(\rho_{s}-\rho\right)}+\frac{1}{\rho_{s}-\rho_{w}}\right. \\
& \left.+\frac{1}{\rho_{s}-\rho}-\frac{K_{g}+\gamma_{\mathrm{dry}}^{2} \rho v_{s}^{2}}{K_{g}\left(\rho_{s}-\rho_{w}\right)+\gamma_{\mathrm{dry}}^{2} \rho v_{s}^{2}\left(\rho_{s}-\rho\right)}\right],
\end{aligned}
$$

$$
\begin{aligned}
\frac{\partial S_{g}}{\partial \rho_{w}} \approx & -S_{g}\left[\frac{K_{w}}{K_{w}\left(\rho_{s}-\rho_{w}\right)-M\left(\rho_{s}-\rho\right)}+\frac{1}{\rho_{s}-\rho_{w}}\right. \\
& \left.+\frac{K_{g}}{K_{g}\left(\rho_{s}-\rho_{w}\right)+\gamma_{\mathrm{dry}}^{2} \rho v_{s}^{2}\left(\rho_{s}-\rho\right)}\right]
\end{aligned}
$$

\section{REFERENCES}

Andreassen, K., K. A. Berteussen, H. Sognnes, K. Henneberg, J. Langhammer, and J. Mienert, 2003, Multicomponent ocean bottom cable data in gas hydrate investigation offshore of Norway: Journal of Geophysical Research, 108, 2399 doi:10.1029/2002JB002245.

Andreassen, K., J. Mienert, P. Bryan, and S. C. Singh, 2000, A double gashydrate related bottom simulating reflector at the Norwegian continental margin: Annals of the New York Academy of Science, 912, 126-135.

Backus, G. E., 1962, Long-wave elastic anisotropy produced by horizontal layering: Journal of Geophysical Research, 67, 4427-4440.

Batzle, M., and Z. Wang, 1992, Seismic properties of pore fluids: Geophysics, 57, 1396-1408.

Beylkin, G., and R. Burridge, 1990, Linearized inverse scattering problems in acoustics and elasticity: Wave Motion, 12, 15-52.

Biot, M. A., and D. G. Willis, 1957, The elastic coefficients of the theory of consolidation: Journal of Applied Mechanics, 24, 594-601.

Bünz, S., and J. Mienert, 2004, Acoustic imaging of gas hydrate and free gas at the Storegga Slide: Journal of Geophysical Research, 109, B04102 doi:10.1029/2003JB002863.

Carcione, J. M., 2001, Wave fields in real media: Wave propagation in anisotropic, anelastic and porous media: Pergamon Press.

Chand, S., T. A. Minshull, D. Gei, and J. M. Carcione, 2004, Elastic velocity models for gas-hydrate-bearing sediments-A comparison: Geophysical Journal International, 159, 573-590.

Collett, T. S., and J. Ladd, 2000, Detection of gas hydrate with downhole logs and assessment of gas hydrate concentrations (saturations) and gas volumes on the Blake Ridge with electrical resistivity $\log$ data, in $\mathrm{C}$. K. Paull, R. Matsumato, P. J. Wallace, and W. P. Dillon, eds., Proceedings of the Ocean Drilling Program, Scientific Results, vol. 164.

Cox, J. L., ed., 1983, Natural gas hydrates: Properties, occurrence and recovery: Butterworth Publishers.

Digby, P. J., 1981, The effective elastic moduli of porous granular rocks: Journal of Applied Mechanics, 48, 803-808.

Domenico, S. N., 1976, Effect of brine-gas mixture on velocity in an unconsolidated sand reservoir: Geophysics, 41, 882-894.

Dvorkin, J., and A. Nur, 1998, Acoustic signatures of patchy saturation: International Journal of Solids and Structures, 35, 4803-4810.

Dvorkin, J., A. Nur, and H. Yin, 1994, Effective properties of cemented granular material: Mechanics of Materials, 18, 351-366.

Dvorkin, J., M. Prasad, A. Sakai, and D. Lavoie, 1999, Elasticity of marine sediments: Rock physics modeling: Geophysics Research Letters, 26, 1781-1784.

Dvorkin, J., and H. Yin, 1995, Contact laws for cemented grains: Implications for grain and cement failure: International Journal of Solids and Structures, 32, 2497-2510.

Ecker, C., J. Dvorkin, and A. Nur, 1998, Sediments with gas hydrates: Internal structure from seismic AVO: Geophysics, 63, 1659-1669. -2000 , Estimating the amount of gas hydrate and free gas from marine seismic data: Geophysics, 65, 565-573.

Frenkel, J., 1944, On the theory of seismic and seismoelectric phenomena in a moist soil: Journal of Physics USSR, 8, 230-241.

Gassmann, F., 1951, Über die Elastizität poröser Medien: Vierteljahrsschrift der Naturforschenden Gesellschaft in Zürich, 96, 1-23.

Geertsma, J., and D. C. Smit, 1961, Some aspects of elastic wave propagation in fluid-saturated porous solids: Geophysics, 26, 169-181.

Guerin, G., D. Goldberg, and A. Meltser, 1999, Characterization of in situ elastic properties of gas hydrate-bearing sediments on the Blake Ridge: Journal of Geophysical Research, 104, 17781-17795.

Hamilton, E. L., 1971, Elastic properties of marine sediments: Journal of Geophysical Research, 76, 579-604.

, 1976, Variations of density and porosity with depth in deep-sea sediments: Journal of Sedimentary Petrology, 46, 280-300.

Helgerud, M. B., 2001, Wave speeds in gas hydrate and sediments containing gas hydrate: A laboratory and modeling study: Ph.D. dissertation, Stanford University.

Helgerud, M. B., J. Dvorkin, A. Nur, A. Sakai, and T. Collett, 1999, Elastic-wave velocity in marine sediments with gas hydrate: Effective medium modeling: Geophysical Research Letters, 26, 2021-2024.

Hill, R., 1963, Elastic properties of reinforced solids: Some theoretical principles: Journal of Mechanics and Physics of Solids, 11, 357-372.

Jacobsen, M., J. A. Hudson, T. A. Minshull, and S. C. Singh, 2000, Elastic properties of hydrate-bearing sediments using effective medium theory: Journal of Geophysical Research, 105, 561-577.

Kaplan, I. R., ed., 1974, Natural gases in marine sediments: Plenum Press

Lee, M. W., D. R. Hutchinson, T. S. Collett, and W. P. Dillon, 1996, Seismic velocities for hydrate-bearing sediments using weighted equation: Journal of Geophysical Research, 101, 20347-20358.

Lodolo, E., A. Camerlenghi, G. Madrussani, U. Tinivella, and G. Rossi, 2002, Assessment of gas hydrate and free gas distribution on the South Shetland margin (Antarctica) based on multichannel seismic reflection data: Geophysical Journal International, 148, 103-119. 
Mavko, G., T. Mukerji, and J. Dvorkin, 1998, The rock physics handbook: Cambridge University Press.

Max, M. D., ed., 2003, Natural gas hydrate in oceanic and permafrost environments: Kluwer Academic Publishers.

Mindlin, R. D., 1949, Compliance of elastic bodies in contact: Journal of Applied Mechanics, 16, 259-268.

Nur, A., G. Mavko, J. Dvorkin, and D. Galmudi, 1998, Critical porosity: A key to relating physical properties to porosity in rocks: The Leading Edge, 17, 357-362.

Paull, C. K., R. Matsumoto, P. J. Wallace et al., 1996, Proceedings of the Ocean Drilling Program, Initial Reports 164.

Paull, C. K., R. Matsumoto, P. J. Wallace, and W. P. Dillon, eds., 2000, Proceedings of the Ocean Drilling Program, Scienific Results vol. 164.

Pride, S. R., and S. Garambois, 2005, Electroseismic wave theory of Frenkel and more recent developments: Journal of Engineering Mechanics, 131, 898-907 doi:10.1061/(ASCE)0733-9399(2005)131:9(898).

Priest, J. A., A. I. Best, and C. R. I. Clayton, 2005, A laboratory investigation into the seismic velocities of methane gas hydrate-bearing sand: Journal of Geophysical Research, 110, B04102 doi:10.1029/2004JB003 259.

Sloan, E. D. Jr., J. Happel, and A. M. Hnatow, eds., 1994, International conference on natural gas hydrates: Annals of the New York Academy of Sciences, 715 .

Spencer, J. W. Jr., M. E. Cates, and D. D. Thompson, 1994, Frame moduli of unconsolidated sands and sandstones: Geophysics, 59, 1352-1361.

Stoll, R. D., 1991, Shear waves in marine sediments-Bridging the gap from theory to field applications, in J. M. Hovem, M. D. Richardson, and R. D. Stoll, eds., Shear waves in marine sediments: Kluwer Academic Publishers, 3-12.
Tinivella, U., 1999, A method for estimating gas hydrate and free gas concentrations in marine sediments: Bolletino di Geofisica Teorica ed Applicata, 40, 19-30.

Tinivella, U., and F. Accaino, 2000, Compressional velocity structure and Poisson's ratio in marine sediments with gas hydrate and free gas by inversion of reflected and refracted seismic data (South Shetland Islands, Antarctica): Marine Geology, 164, 13-27.

Tinivella, U., and E. Lodolo, 2000, The Blake Ridge bottom-simulating reflector transect: Tomographic velocity field and theoretical model to estimate methane hydrate quantities, in C. K. Paull, R. Matsumoto, P. J. Wallace, and W. P. Dillon, eds., Proceedings of the Ocean Driling Program, Scientific Results, 164

Walton, K., 1987, The effective elastic moduli of a random packing of spheres: Journal of the Mechanics and Physics of Solids, 35, 213-226.

Wang, Z., and A. Nur, 1992, Elastic wave velocities in porous media: A theoretical recipe, in Z. Wang, and A. Nur, eds., Seismic and acoustic velocities in reservoir rocks, vol. 2-Theoretical and model studies: SEG.

Winkler, K. W., 1983, Contact stiffness in granular porous materials: Comparison between theory and experiment: Geophysical Research Letters, 10, 1073-1076.

Zillmer, M., E. R. Flueh, and J. Petersen, 2005a, Seismic investigation of a bottom simulating reflector and quantification of gas hydrate in the Black Sea: Geophysical Journal International, 161, 662-678 doi:10.1111/ j.1365-246X.2005.02635.x.

Zillmer, M., T. Reston, T. Leythaeuser, and E. R. Flueh, 2005b, Imaging and quantification of gas hydrate and free gas at the Storegga slide offshore Norway: Geophysical Research Letters, 32, L04308, doi: 10.1029/ 2004 GL02153. 